\title{
The Impact of Food Allergy Training on a Teacher's Self- Efficacy when Managing Food Allergic Students
}

\author{
Paige Cook ${ }^{1}$
}

'Lane Technical High School, Chicago, Illinois, USA

\section{$\underline{\text { ABSTRACT }}$}

Food allergy training for teachers remains an important, but commonly overlooked, aspect of education as millions of children have been diagnosed with food allergies. Therefore, it is pertinent teachers receive food allergy training that is most beneficial in growing a teacher's understanding for teachers to be best suited in correctly helping food allergic students. Four food allergy training methods: hands-on in-person (actual handling of epinephrine), non-hands in-person, group video, and individual video, and their effects on the self-efficacy of participants is the focus of this study. Teachers were contacted from within the city of Chicago, both public and private, and from a mixture of food allergy training methods and were administered an online survey which asked questions pertaining to their food allergy knowledge, perception, and self-efficacy. Participants were given statements to which they would respond on a fivepoint Likert scale, scored using a chi-square test. Ultimately, the hypothesis that teachers who engaged in hands-on in-person would showcase higher levels of self-efficacy, due to higher levels of engagement, was not supported. While several statements proved significant after data analysis, there was not enough significance to prove a meaningful relationship between the self-efficacy and training method.

\section{Introduction}

\section{Background}

Currently, it is estimated that 32 million Americans, including 5.6 million children, have been diagnosed with food allergies (FARE, n.d.). A food allergy is defined as an immunological response wherein the immune system attacks a food protein, called allergens, that is not usually considered harmful (Lee, Kwon, \& Sauer, 2016). The eight most common allergens in the United States are: milk, eggs, fish, crustacean shellfish, wheat, soy, peanuts, and tree nuts. These eight allergens account for 90 percent of all food allergies (John Hopkins Medicine, n.d.). The occurrence of food allergies has greatly risen in the last few decades, increasing 50\% between 1997-2011 (CDC, 2020). Certain allergens are rising at higher rates than others, as peanuts and tree nut allergies have appeared to triple since 1997.

The severity of an allergic reaction depends upon an individual's allergy. Peanuts, tree nuts, fish, and shellfish are known to cause the most severe reactions (John Hopkins Medicine, n.d.). Some individuals will have mild to moderate reactions after ingesting an allergen, which can manifest in the forms of rashes, tingling hands, and gastrointestinal issues. However, in severe cases, individuals who ingest an allergen can experience anaphylaxis, also known as anaphylactic shock (Lee, Kwon, \& Sauer, 2016). Once anaphylaxis begins, the only treatment is the drug epinephrine, commonly known as adrenaline. Treatment needs to be administered within minutes of the onset of systems and may require more than one dose (FARE, n.d.). Without proper treatment, anaphylaxis can cause death within 15 minutes (MedlinePlus, 2019). For children, visits to the emergency room for anaphylaxis have more than doubled from 2009 to 2013 (Faarbman \& Michelson, 2017).

As children spend a significant portion of their day and year at school, it is important that food allergic children are able to receive the help they require. Within schools, it is estimated that about 1 in 13 children, or two to three per classroom, has a food allergy (CDC, 2019). Additionally, 15 percent of school-aged children have had an 
allergic reaction while at school, which for many is the first allergic reaction that the child has experienced (FARE, n.d.). Therefore, nurses, teachers, and staff of each school are expected to properly manage food allergies and anaphylaxis for students (Tsuang et al., 2018). Presently, the United States has voluntary guidelines for schools and education programs on how to properly manage student's food allergies. Illinois has enacted state guidelines for managing allergies, which require that food allergy training for staff should be conducted every other year with anaphylaxis drills occurring yearly (Szychlinski et al., 2015).

It is possible that different types of allergy training will lead to changes in a teacher's self-efficacy - an individual's belief that they have the ability to execute necessary and proper behaviors in particular situations, such as administering epinephrine during an allergic reaction. Self-efficacy has been shown to be a reliable measure for estimating a teacher's ability to properly respond to allergic reactions (Polloni et al., 2016). Therefore, this study focuses on comparing four different types of food allergy training methods to determine which method correlates with the highest rate of self-efficacy in teachers when managing food allergic students.

\section{Literature Review}

\section{Perception of Food Allergies}

The stark increase of allergy rates in recent years has led to a growth in food allergy research and experiments, resulting in a sizable existing body of research on the matter. Numerous studies have been done on the general knowledge of food allergies. Lyons and Forde (2004) researched the extent of people's perceptions toward others who have food allergies by surveying 162 students at the University of Birmingham using a 5-point Likert scale. The study concluded that people without food allergies believed food allergies posed a larger impact on daily life than the food allergic participants rated it, however, both showed a low understanding in food allergy terms, such as anaphylaxis. Similarly, Majowicz, Jung, Courtney, and Harrington (2017) attempted to determine whether there was a link between the perceived risk of food allergies and age in Ontario, Canada from age 13 to 24 . The overall trend appeared to be that the older the participant, the more unconcerned became their perception of food allergies, likely due to these participants being more self-reliant. Other factors that affected concern was if the participant was female, or if they had worked within a setting that handles food, such as an educational setting. These groups were believed to have a higher rate of concern due to having more experience with food allergies and likely seeing the effects of an allergy gone mistreated or mishandled.

Additionally, studies have been conducted that focus on the perceptions of food allergies from people in a close relationship with the affected, rather than the general population's concern regarding food allergies. Sanagavarapu (2018) focused heavily on the emotional and mental toll that food allergic children take on their mother, specifically focusing on the child's transition to preschool. Unlike many studies, Sanagavapu utilized a qualitative design and focused on a select group of 10 mothers from different Australian schools due to Australia's high rate of food allergies. The themes of the study were recorded and categorized upon completion. $70 \%$ found the transition to school to be emotionally stressful and challenging while all agreed that keeping their child safe would be an ongoing challenge (Sanagavapu, 2018). Major barriers to a smooth transition to the school setting were the school's lack of communication, transitional planning, lapses in food allergy management procedures, and a lack of parental involvement. Therefore, the researcher concluded that there is a major discontinuity between parents and the school. The ongoing discontinuity is something vital that needs to be addressed to prevent the prevalent emotional stress in mothers and their food allergic children. Another study that focused specifically on the caregivers was a cross-sectional Japanese study that compared the responses from caregivers of food allergic children to those of non-food allergic children caregivers, all caregivers of children from first to sixth grade (Yamato-Kiwako, 2015). The non-food allergic caregivers were split between two groups: never having witnessed a reaction and having witnessed a food reaction. Caretakers that had witnessed a reaction showed considerably more interest overall in learning more about allergies, generally. The first group was considerably less confident in their abilities to correctly manage a FA, perhaps revealing that it 
benefits a caretaker to have some knowledge of a food allergy to properly manage it. Actually having witnessed a reaction, without having any previous caretaking knowledge, appears to make a caretaker more confident in their own actions (Yamato-Kiwako, 2015). Therefore, having previous knowledge of a food allergy could help a caretaker while in an educational setting.

\section{Self-Efficacy}

Yamato-Kiwako's study on caregiver's confidence translates into the food allergy studies on self-efficacy, shifting the focus on confidence from an external force to an internal one. Jacobson, Vale, Sambell, and Devine (2018) derived a 41-question study focusing on teacher allergy readiness by measuring teacher confidence in their allergy implementation. It was found that the most common group to partake in anaphylaxis training was educators, staff members, and directors or coordinators. Some barriers to training were found to hours of operation of school, cost of training, and a lack of face-to-face training. The largest obstacle to overcome is the apathy displayed by parents of non-allergic children, as many are unsupportive of implementing more measures. These parent's unwillingness only highlights the need for the additional spread of food allergy information. Due to these barriers, educators hold a large concern regarding managing anaphylactic shock within their student body, causing the majority of staff to wish they had more training, signifying that they are unprepared to handle all cases of anaphylaxis (Jacobson, Vale, Sambell, \& Devine, 2018). Educator's confidence in themselves rose after they had allergy training. The study focused primarily on Australia, which has one of the highest country rates of food allergies for children, and if they have such difficulties within their schools, it may signal the need for food allergy training for global schools overall.

Similarly, Yamamato-Hanada and Ohyra (2018) conducted a cross-sectional study on Japanese public-school teachers in Tokyo to investigate the factors that are associated with self-efficacy for educators. The researchers acknowledge that building self-efficacy is vital to improving both disease and food allergy management, yet, the factors associated with building self-efficacy are not completely identified. They measured self-efficacy by measuring the 828 teacher responses on a five-point Likert scale to the statement 'I am confident in taking appropriate action if I see a child experiencing an adverse response due to a food allergy' while taking other factors such as demographics and personal experiences into account (Yamamato-Hanada \& Ohyra, 2018). The range of data supports their idea of self-efficacy being promoted by a variety of factors. The most beneficial factor being the duration of a teacher's career, and whether they hold/or have held a managerial position. Additionally, teachers that had partaken in a food allergy training session displayed higher rates of self-efficacy than teachers who had not, which previous studies such as Polloni's (2016) and Yamato-Kiwako's (2018) have indicated. A final factor to boosting self-efficacy is having simulation-based training — teachers have hands-on experience of responding to a food-allergy — allowing teachers to gain insight into the stress and fast-paced aspect of an actual allergic reaction. Overall, the results promote the idea that self-efficacy is something that can be affected in teachers, especially determined by their type of food allergy training (Yamamato-Hanada \& Ohyra, 2018). Polloni (2016) similarly found that many aspects determine self-efficacy. In a survey of 440 Italian teachers and school caretakers, self-efficacy was a constantly varying factor. Teachers and caretakers revealed higher rates of self-efficacy when working with other school professionals but displayed lower rates of self-efficacy when asked to manage anaphylaxis. Another concern was guaranteeing full participation for food allergic students in all school activities. $66 \%$ of participants who had previously worked with food allergic students did display a slightly increased self-efficacy, however, their rate of anaphylaxis self-efficacy specifically remained low (Polloni, 2016). The disparity between managing food allergies and managing anaphylaxis for self-efficacy builds the conclusion that school personnel food allergy confidence is lacking. The lack of knowledge could easily be addressed through school's promoting allergy training to a higher degree or simply working on the staff's specific areas that need improvement.

\section{Training}

Furthermore, many researchers studied the increase in self-efficacy for teachers before and after administered food allergy training sessions. Tsuang et al. (2018) assessed knowledge, attitude, and confidence levels regarding food 
allergy and anaphylaxis management, which was administered to nurses who were asked to repeat the survey every six months throughout the two years after the session. After training, the nurse's knowledge score always remained higher than the pre-training score of $77 \%$. Attitude increased from $78 \%$ to $85 \%$; confidence increased from $81 \%$ to $86 \% .18 \%$ of participants scored a perfect score after training, however, the lowest confidence score remained on recognizing symptoms of anaphylaxis (Tsuang et al., 2018). Although confidence in recognizing anaphylaxis symptoms remained low, the results illustrate that training sessions have positive, lasting effects on knowledge, attitude, and confidence. Information learned from training sessions can prevail if staff keeps up with refreshing themselves on the information, as the nurses did every few months with the survey. Gonzalez-Mancebo (2018) analyzed teachers, cooks, dining-room monitors, and summer-camp leaders from a food allergy training session to monitor their rates of self-efficacy. As with Tsuang et al. (2018), as a whole, the mean score for all eight tested concepts of food allergy training improved significantly before and after training. 90.9\% received correct answers after training (GonzalezMancebo, 2018). Interestingly, participants were presented with a clinical case where they had to choose, out of four options, the best course of action to treat the 'child', emphasizing a simulation-based experience. Participants' knowledge appeared to depend on situations that were more directly related to their jobs.

\section{Gap}

While there exist large amounts of research on the extent of food allergy knowledge and self-efficacy of staff before and after allergy training sessions, there is at present a lack of research comparing different methods of allergy training to each other. No study has taken staff from different training sessions and compared their feelings of self-efficacy and food allergy knowledge to determine if one appears more beneficial than the others. Additionally, hands-on training is the food allergy training that has been emphasized the most through research, revealing another gap. Other training methods, such as videos, have been neglected; how they could impact a teacher's self-efficacy remains unknown. This study will address the two gaps by finding teachers with four different methods of training and comparing the four to each other.

\section{Hypothesis}

Due to information gained from previous researcher's studies, I hypothesize that participants who partake in handson training will display the highest rates of self-efficacy compared to participants who partook in non-hands-on and video training methods. According to Yamamato-Hanada and Ohrya (2018), simulation based, or hands-on, training can affect a teacher's self-efficacy in a positive manner. Participants with hands-on training will better understand the stress associated with dealing with a food allergy reaction and will have more insight than other participants in how much time and resources they have during a reaction. Given that there is also an instructor present in the room for hands-on training, participants would have had the opportunity to ask any questions or gain clarity, something that is lacking in group and individual video participants. None but hands-on participants have had the opportunity to handle an Epi-Pen, already knowing how to administer medicine, likely lowering the stress of having to handle new equipment in the case of a reaction as with the other allergy training groups.

\section{Methodology}

\section{Overview}

Data was collected by using a cross-sectional study in the form of a quantitative survey utilizing short answers, dropdown questions, and a five-point Likert scale in which a participant rates their opinion on each provided statement. Each participant was administered the same online survey that consisted of questions regarding demographics, food allergy knowledge, and food allergy training and perception that ultimately measures self-efficacy. Participants were 
gathered through purposive sampling as the entire sample consisted of teachers. Additionally, the survey was voluntary response as each participant received an email which clearly outlined their choice in opening and participating in the survey. A consent form was provided at the beginning of the survey and participants had the ability to withdraw at any time. Upon completion, participants were given the option to supply their own email to be debriefed at the conclusion of data analysis. This study was reviewed and approved by an Institutional Review Board (see Appendix A), ensuring the conduct of the survey was done in an ethical manner due to the participation of human subjects.

\section{Participants}

The participants were all teachers from Chicago, Illinois. To gain access to the population, the principal of each school was first contacted through email. Principals were provided with information that detailed the purpose of the study in order to gain informed consent from them that their school may be surveyed. Schools were contacted throughout the city of Chicago regardless of neighborhood factors, such as ethnicity or wealth, and type of school (ex. elementary, public, private). Furthermore, teachers from all specializations were contacted, no matter what age group they taught or length of teaching career. Given that the survey only focuses on types of food allergy training, it does not matter what age range a teacher primarily teaches as the type of food allergy training will still be uniform throughout the school. Similarly, no matter what area of the city a school is located, it is still mandated for each school to have some type of food allergy training. Another aspect of utilizing all teachers regardless of specialization was to provide more resources and opportunity to gather diverse data in all four food allergy training categories. Given that Chicago Public Schools has uniform allergy training throughout the entire district, it was necessary to contact private schools to provide more than one type of allergy training.

After gaining consent from the head of school, all teachers were contacted through email that detailed the purpose of the study and reaffirmed that they give their consent to partaking in it.

\section{Creation of Tools}

Self-efficacy was selected as the dependent variable as perceived self-efficacy is something thought to be changed by allergy training method (Polloni, 2016). Eight questions on the survey measured self-efficacy in participants. Each question began with the stem "I believe..." followed by a statement regarding a teacher's confidence that was to be rated from one to five on a Likert scale. One was equated to strongly disagree, five being strongly agree, and three being the neutral choice. The self-efficacy statements were modelled off questions created by Polloni et. al in their 2016 study. The 2016 questionnaire was developed by a multidisciplinary team of experts that was based on a combination of previous research and Bandura's guidelines for creating self-efficacy scales. The questionnaire was given to a target audience to test the level of clarity before being utilized in the study meaning it is an understandable and tested method of properly measuring self-efficacy.

Additional questions on the survey were self-created, however, they still modelled Polloni's survey, along with other food allergy studies, in asking questions about demographics and specific types of food allergy training. The demographic section consisted of one short answer and five drop-down questions. The short answer questions asked the years of teaching experience, mandated that the answer be given in increments of .5 to provide continuous data, while the drop down questions inquired on department, teacher's own food allergy status, close relation's food allergy status, and whether or not the participant had responded or witnessed an allergic reaction in or out of the classroom. Gathering general knowledge about the teacher's partaking in the survey is vital for accounting for possible outliers in the data. For example, a health teacher may be more likely to understand food allergies and their training than a history teacher due to a difference in prior experience on food allergies. More so, a teacher with a confirmed food allergy already knows how to respond to a reaction, from their personal experience, accounting for their high levels of knowledge throughout the survey.

One drop-down and 11 Likert-scale questions were utilized to analyze a teacher's confidence in their type of food allergy training. Participants were first required to choose their last method of food allergy training. The four types included were: hands-on in-person, hands-off in-person, group video, and individual video. Hands-on and non- 
hands-on both required the presence of an instructor; however, a requirement of hands-on training was that a participant must have handled an Epi-Pen (epinephrine). Group video and individual video had no instructor, rather, the participant watched a video for their food allergy training, either alone (individual) or with other teachers (group). Using their type of food allergy training, participants were presented with 11 five-point Likert scale questions, corresponding to the same scale as the self-efficacy questions beginning with the question stems: 'During training, I was' (2), 'I feel' (6), 'During training, I understood' (1), 'During training, I retained' (1), and 'I would' (1). These questions were asked to fully understand the difference in types of food allergy training as one may allow teachers to better retain and understand the information provided to them than another. It is thought that participants with a higher level of understanding regarding their food-allergy training will have higher rates of self-efficacy. The survey concluded with the eight questions on self-efficacy.

\section{Data Calculation}

The data was organized in three ways, the first to test the hypothesis and the remaining two to test for possible confounding variables. To test the hypothesis, the data was organized in four categories: group video, hands-on in-person, individual video, and non-hands-on in-person. For the confounding variables, in one test the participants were organized by department, excluding physical education due to having one respondent, and, in the second test, was organized by years of experience. A Chi-Square Test, a method used to interpret categorical variables, was utilized to analyze the data given that it was categorical in the form of a Likert scale. The significance value for the test was selected as $\boldsymbol{\alpha}=0.05$ meaning the data is only significant with a p-value of less than .05 . The Chi-Square observes how likely a distribution is due to chance, one not being due to chance having a p-value of less than .05. For each question, a Chi-Square was made with the columns: strongly disagree, disagree, neutral, agree, and strongly agree, with the rows either being methods, department, or years. The chi-squared value and p-value were recorded for each question to which the results were organized into three tables.

\section{Findings}

Out of the 74 teachers that responded to the survey, 2 (2.7\%) experienced a group video training, $36(48.6 \%)$ handson in-person, 28 (37.8\%) individual video, and 8 (10.8\%) non hands-on in-person. Table 1, below, shows the results for the Chi-square test run when dividing participants into their respective food allergy training method. Four instances reveal significant differences between training methods. All four statements that yielded significant results stemmed from the questions regarding each teacher's perception of their training. The first instance is 'I feel my school's allergy training is informative," having a p-value of .00453. All hands-on in-person participants answered the question neutrally (2), or with regular or strong agreement (34). All other groupings had at least one participant answer the question negatively, individual video having 1 participant strongly disagree with the statement. The second instance is "I feel my school's food allergy training is up-to-date," with a p-value of .003644. Similarly, all teachers undergoing handson in-person training answered the question with agreement, however, all other groups revealed at least one participant with negative views. The third statement is "I feel my school's training taught me how to properly respond to a reaction," with a p-value of .002756. The final significant statement is, "I feel my school provides the proper equipment to manage food allergies," having a p-value of .00188. As with the first two statements, hands-on in-person participants all answered neutral or with agreement, majority leaning towards strong agreement. 


\begin{tabular}{|c|c|c|}
\hline Question & $x^{2}$ & $p$ \\
\hline I know what the definition of a food allergy is & 13.5735 & 0.3288 \\
\hline I know what anaphylaxis is & 13.799 & 0.3137 \\
\hline I know how to treat anaphylaxis & 14.6636 & 0.2603 \\
\hline I know the top eight food allergens & 2.4513 & 0.9983 \\
\hline I know how to handle an Epi-Pen & 15.0375 & 0.2394 \\
\hline I know how to recognize anaphylaxis symptoms & 8.797 & 0.7202 \\
\hline During training, I was taught what a food allergy and/or anaphylaxis is & 17.1908 & 0.1426 \\
\hline During training, I was paying attention & 10.7122 & 0.5534 \\
\hline I feel my school's food allergy training is informative & 28.5904 & 0.00453 \\
\hline I feel my school's training is up to date & 29.2283 & 0.003644 \\
\hline I feel anxious when thinking about managing food allergies & 5.0204 & 0.9573 \\
\hline I feel my school's training taught me how to properly respond to a reaction & 30.0375 & 0.002756 \\
\hline I feel confident in my abilities to manage food allergies & 16.5901 & 0.1657 \\
\hline I feel my school provides the proper equipment to manage food allergies & 31.1329 & 0.00188 \\
\hline During training, I understood the information taught to me & 16.4877 & 0.1699 \\
\hline During training, I retained the information taught to me & 20.1269 & 0.0647 \\
\hline I would like to have more food allergy training & 10.3348 & 0.5866 \\
\hline I believe food allergy training is important to know & 9.4377 & 0.6652 \\
\hline I believe food allergies pose a risk to my students & 9.9542 & 0.62 \\
\hline I believe I can assure a safe setting for my students & 9.952 & .6200 \\
\hline I believe I can work with families/co-workers to create a safe setting & 7.4008 & 0.83 \\
\hline $\begin{array}{l}\text { I believe... [I can guarantee full participation in school activities for food al- } \\
\text { lergic students] }\end{array}$ & 7.6645 & 0.81077 \\
\hline I believe I can recognize anaphylaxis symptoms & 9.045 & 0.6991 \\
\hline I believe I can manage allergens avoidance (reading labels, etc.) & 7.1502 & 0.8475 \\
\hline $\begin{array}{l}\text { I believe I can administer allergy treatment to a student having a sudden re- } \\
\text { action }\end{array}$ & 11.6923 & 0.4707 \\
\hline
\end{tabular}


13 (17.5\%) participants worked five or less years, 17 (23\%) participants worked 6-10 years, 17 (23\%) participants worked 11-15 years, and the remaining 27 (36.7\%) had over 16 years of teaching experience. No statements tested for p-values of less than 0.05 . Table 2 , below, shows the corresponding p-values for each statement. The statement with the lowest p-value was, "I feel my school's training taught me how to properly respond to a reaction," at a p-value of .1011. Similar to the food allergy training test, this statement came from the perception section of the survey. More teachers with 1-5 years of teaching experience responded with disagreement (5) than the other three groups combined. Majority of teachers responded to the statement with agreement.

Table 2. Chi-Squared Test for years of teaching experience

\begin{tabular}{|c|c|c|}
\hline Question & $x^{2}$ & $p$ \\
\hline I know what the definition of a food allergy is & 8.2828 & 0.7627 \\
\hline I know what anaphylaxis is & 4.0027 & 0.9834 \\
\hline I know how to treat anaphylaxis & 8.9488 & 0.7073 \\
\hline I know the top eight food allergens & 13.3108 & 0.3468 \\
\hline I know how to handle an Epi-Pen & 4.6219 & 0.9694 \\
\hline I know how to recognize anaphylaxis symptoms & 7.9637 & 0.7879 \\
\hline During training, I was taught what a food allergy and/or anaphylaxis is & 7.5069 & 0.8224 \\
\hline During training, I was paying attention & 5.9038 & 0.9209 \\
\hline I feel my school's food allergy training is informative & 15.0621 & 0.2381 \\
\hline I feel my school's training is up to date & 9.949 & 0.6204 \\
\hline I feel anxious when thinking about managing food allergies & 6.0755 & 0.9122 \\
\hline I feel my school's training taught me how to properly respond to a reaction & 18.5094 & 0.1011 \\
\hline I feel confident in my abilities to manage food allergies & 6.9265 & 0.8624 \\
\hline I feel my school provides the proper equipment to manage food allergies & 8.0952 & 0.7776 \\
\hline During training, I understood the information taught to me & 5.941 & 0.919 \\
\hline During training, I retained the information taught to me & 14.752 & 0.2553 \\
\hline I would like to have more food allergy training & 7.8195 & 0.7991 \\
\hline I believe food allergy training is important to know & 5.9667 & 0.9178 \\
\hline I believe food allergies pose a risk to my students & 6.355 & 0.8971 \\
\hline I believe I can assure a safe setting for my students & 9.7058 & 0.64175 \\
\hline I believe I can work with families/co-workers to create a safe setting & 1.7139 & 0.9997 \\
\hline $\begin{array}{l}\text { I believe... [I can guarantee full participation in school activities for food aller- } \\
\text { gic students] }\end{array}$ & 6.682 & 0.8777 \\
\hline I believe I can recognize anaphylaxis symptoms & 9.743 & 0.6385 \\
\hline
\end{tabular}


Table 2 continued.... Chi-Squared Test for years of teaching experience

I believe I can manage allergens avoidance (reading labels, etc.)

I believe I can administer allergy treatment to a student having a sudden reaction

\subsection{3}

0.9505

Note. $\mathrm{N}=25$. Significant at $\mathrm{p}<0.05$.

Of the 74 participants, $19(25.7 \%)$ were in history, $7(9.5 \%)$ in fine arts, $26(35.1 \%)$ other, $1(1.4 \%)$ physical education, $12(16.2 \%)$ science, and $9(12.2 \%)$ in social science/history. No participants considered themselves to be in computer science or driver's ed. For the chi-square test, P.E was omitted due to only having 1 response, meaning no comparison of data with another similar teaching could be made. Similarly, as the test for years of experience, there were no significant statements when comparing teachers only regarding their department. Each statement revealed even higher p-values than are seen in Table 2, all being over .5, largely over .05 to be significant.

Table 3. Chi-Squared Test for teaching department

\begin{tabular}{|l|l|l|}
\hline Question & x2 & $\boldsymbol{p}$ \\
\hline I know what the definition of a food allergy is & 5.7087 & 0.9909 \\
\hline I know what anaphylaxis is & 10.1712 & 0.8575 \\
\hline I know how to treat anaphylaxis & 15.2304 & 0.5078 \\
\hline I know the top eight food allergens & 14.7607 & 0.5422 \\
\hline I know how to handle an Epi-Pen & 9.4177 & 0.8952 \\
\hline I know how to recognize anaphylaxis symptoms & 11.1659 & 0.7991 \\
\hline During training, I was taught what a food allergy and/or anaphylaxis is & 9.6624 & 0.8837 \\
\hline During training, I was paying attention & 10.3689 & 0.8467 \\
\hline I feel my school's food allergy training is informative & 10.4303 & 0.8432 \\
\hline I feel my school's training is up-to-date & 14.5242 & 0.5597 \\
\hline I feel anxious when thinking about managing food allergies & 10.0528 & 0.8639 \\
\hline $\begin{array}{l}\text { I feel my school's training taught me how to properly respond to a re- } \\
\text { action }\end{array}$ & 10.3257 & 0.8491 \\
\hline $\begin{array}{l}\text { I feel confident in my abilities to manage food allergies } \\
\text { I feel my school provides the proper equipment to manage food aller- } \\
\text { gies }\end{array}$ & 12.4112 & 0.715221 \\
\hline During training, I understood the information taught to me & 8.8885 & 0.2612 \\
\hline During training, I retained the information taught to me & 11.2324 & 0.9179 \\
\hline I would like to have more food allergy training & 9.2719 & 0.7949 \\
\hline I believe food allergy training is important to know & 4.7564 & 0.901794 \\
\hline & & 0.9968 \\
\hline
\end{tabular}




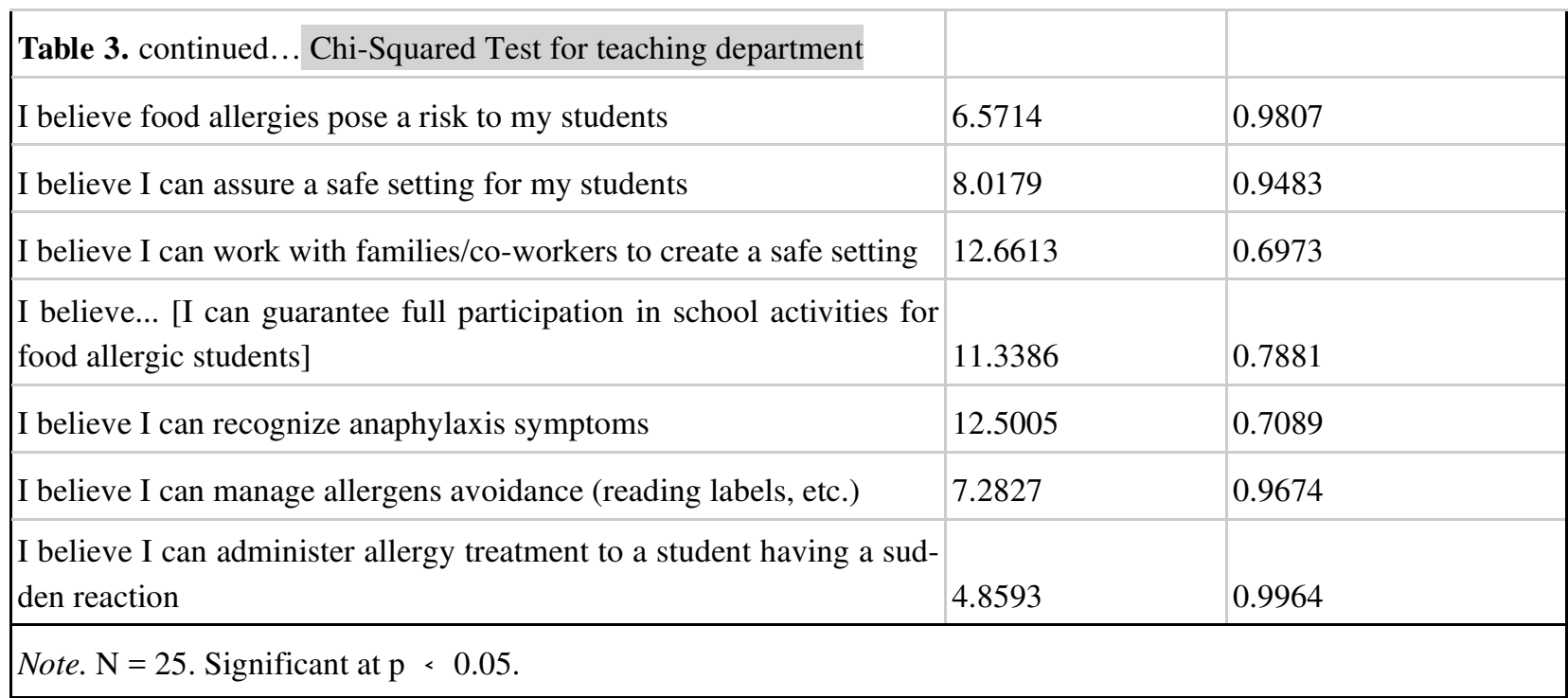

\section{Discussion}

The hypothesis that participants who partook in hands-on in-person allergy training will showcase higher levels of self-efficacy than the other methods of allergy training was not supported based on the study's results. While four questions were significant during the allergy methods chi-square, as they had p-values of less than .05 , there were 25 questions overall, meaning that only $16 \%$ of the questions showed any meaningful relationship between allergy training and a participant's self-efficacy. The minority of questions reveal significance, not creating enough data to support a meaningful relationship between the two variables. Additionally, the four questions that showed significance focused more heavily on how a teacher perceived their food allergy training rather than a teacher's perception of their own abilities resulting from the food allergy training. None of the questions that specifically related to self-efficacy, modified from Polloni's 2016 study, revealed any drastic change. Therefore, it cannot be said that there is enough difference between the groups, the numbers being too close. In accounting for possible outliers with the second and third chi-square and comparing table 1 with table 2 and 3, there is not also not a large enough difference between the groups. Many p-values are only tenths or hundredths apart from each other, even though the p-values are higher in table 2 and 3 than in table 1 . Simply looking at a teacher's perception on training allergy management, not looking at how it affects a teacher's perception on them self, it supports that different allergy training methods do cause a difference in certain aspects of a teacher's thoughts. However, the difference does not pertain to self-efficacy, at least to a noticeable degree, and the hypothesis of there being a change in self-efficacy is ultimately not supported by the gathered data.

\section{Limitations}

A major limitation to the study was the low number of participants. While there were 74 participants, majority partook in hands-on in-person or individual video allergy training. More participants from hands-off in-person or group video training would have allowed for a better comparison of the data and a better relationship drawn between self-efficacy and food allergy training. Another limitation is the diversity of teacher types. Some departments had no representation, such as computer science and driver's ed, meaning the data does not accurately represent schools. Additionally, there was a single physical education teacher, not allowing that department to be compared either. 26 participants considered themselves as 'other'. 'Other' could be replaced by a more detailed listing of departments, such as by adding foreign 
language or teaching assistants. 'Other' makes it unknown whether or not there is a correlation between department and self-efficacy due to the ambiguity of the term.

\section{Implications and Future Directions}

While the hypothesis was not proven in this study, the focus and aim of the study still prove important as it is one of the first to begin investigating the relationship between teacher's self-efficacy and food allergy training methods. Using a wider range of participants from each of the four categories and making sure that each category has a more equal number of participants would ensure more accurate data to be analyzed. The data, if proven significant, could be utilized to determine the best method of food allergy training to train teachers which, therefore, could be used to modify current guidelines or training processes at many schools.

Future variations of the study could utilize a pre-test methodology. Doing pre-test post-test would quickly reveal the extent that food allergy training affects self-efficacy, eliminating many external variables. Additionally, this study could be conducted as a longitudinal study in following the same teachers over many years of their career. Such a study would allow for an examination of food allergy training type effect on self-efficacy and the effect years of experience has on self-efficacy. Any changes in self-efficacy level would also be measurable, revealing the effect that time has on self-efficacy. Eventually, the study could be generalized to a larger population than Chicago, in which many teachers have the same food allergy training due to CPS guidelines. Utilizing different regions may introduce new prevalent training methods that may prove better in promoting self-efficacy than the one's observed in this study.

\section{References}

Allergic reactions. (n.d.). MedlinePlus. Retrieved May 20, 2020, from medlineplus.gov/ency/article/000005.htm

Farbman \& Michelson. (2016). Anaphylaxis in children. Current Opinion in Pediatrics, 28(3), 294-297.

doi: $10.1097 /$ MOP.0000000000000340

Food allergies. (2020). Centers for Disease Control and Prevention. Retrieved May 20, 2020, from

www.cdc.gov/healthyschools/foodallergies/index.htm

Food allergies in children. (n.d.). John Hopkins Medicine. Retrieved December 8, 2019, from

www.hopkinsmedicine.org/health/conditions-and-diseases/food-allergies-in-children

Gonzalez-Mancebo, E.,et al. (2018). Analysis of the effectiveness of training school personnel in the management of food allergy and anaphylaxis. Allergologia et Immunopathologia, 60-63. doi:10.1016/j.aller.2018.05.005

Lee, Y. M., Kwon, J., \& Sauer, K. (2016). Child nutrition professional's attitudes, perceived challenges, and training relating to food allergies. Health Behavior \& Policy Review, 3(2), 165-175.

Lyons, A. C., \& Forde, E. M. E. (2004). Food allergy in young adults: Perceptions and psychological effects. Journal of Health Psychology, 9(4), 497-504. doi:10.1177/1359105304044032

Majowicz, S. E., Jung, J. K. H., Courtney, S. M., \& Harrington, D. W. (2017). Exploring the concern about food allergies among secondary school and university students in Ontario, Canada: A descriptive analysis. Journal of Allergy, 2017, 1-8.doi:10.1155/2017/2051916 
Polloni, L. et al. (2016). School personnel's self-efficacy in managing food allergy and anaphylaxis. Pediatric Allergy \& Immunology, 27(4), 356-360. doi:10.1111/pai.12550

Sanagavarapu, P. (2018). Experiences and support needs of mothers of children with food allergy during the transition to school. Early Childhood Education Journal, 46(5), 523-534. doi:10.1007/s10643-017-0880-8

Szychlinski et. al.(2015). Food allergy emergency preparedness in Illinois schools: Rural disparity in guideline implementation. The Journal of Allergy and Clinical Immunology: In Practice, 3(5), 805-807.

doi:10.1016/j.jaip.2015.04.017

Tsuang, A., Atal, Z., Demain, H., Patrick, K., Pistiner, M., \& Wang, J. (2018). Benefits of school nurse training sessions for food allergy and anaphylaxis management.

The Journal of Allergy and Clinical Immunology: In Practice. 1-3. doi:10.1016/j.jaip.2018.05.015

What is a food allergy? (n.d.). FARE. Retrieved December 8, 2019, from www.foodallergy.org/resources/what-food-allergy

Yamato-Kiwako, K, et al. (2015). Caregivers of children with no food allergy-- Their experiences and perception of food allergy. Pediatric Allergy \& Immunology, 26(7), 614-617. 